\title{
El ultrasonido pulmonar en la evaluación del síndrome alveolo-intersticial en el postoperatorio de cirugía valvular aórtica e hipertensión pulmonar
}

\author{
Pulmonary ultrasound in the evaluation of the alveolar-interstitial syndrome in the \\ postoperative period of aortic valve surgery and pulmonary hypertension
}

\section{Luis E. Santos-Martínez ${ }^{1,2 *}$, Jessica Garduño-López¹, Gastón Mendoza-Copa¹, Edgar García-Cruz', Efren Melano-Carranza1 ${ }^{1}$, Miguel Á. Hernández-Márquez ${ }^{1}$ y Francisco M. Baranda-Tovar ${ }^{1}$}

${ }^{1}$ Departamento de Cuidados Intensivos Posquirúrgicos Cardiovasculares, Secretaría de Salubridad y Asistencia, Instituto Nacional de Cardiología Ignacio Chávez; ${ }^{2}$ Departamento de Hipertensión Pulmonar y Corazón Derecho, Instituto Mexicano del Seguro Social, Centro Médico Nacional Siglo XXI, Unidad Médica de Alta Especialidad Hospital de Cardiología, Ciudad de México, México

La evaluación pleuropulmonar en las unidades de cuidados intensivos $(\mathrm{UCl})^{1-4}$ tradicionalmente se realiza con radiografía $(\mathrm{Rx})$ de tórax simple en vista anteroposterior. Su limitación ha sido la baja precisión diagnóstica en la enfermedad pulmonar. La tomografía axial del tórax resolvió este problema, pero su inconveniente fue la radiación y que se requiere trasladar al paciente fuera de la $\mathrm{UCI}^{1-4}$.

El ultrasonido pulmonar (USG-P) ha complementado esta evaluación ${ }^{1}$. Su utilidad en la UCl se centra en el diagnóstico de los síndromes de consolidación pulmonar, derrame pleural, edema pulmonar (EP) y neumotórax ${ }^{1-4}$; sin embargo, en la evaluación del EP no parece discriminar entre el origen cardiogénico ${ }^{3}$ y no cardiogénico ${ }^{5}$, por lo que en el ultrasonido se le ha denominado síndrome alveolo-intersticial.

En el postoperatorio temprano de cirugía cardíaca existe la posibilidad de que el paciente curse con EP cardiogénico $0^{6} \mathrm{y} / 0$ no cardiogénico de causas diversas ${ }^{5}$, como la infección pulmonar o la lesión pulmonar aguda por transfusión (TRALI)?
Presentamos la evolución clínica, imagen, hemodinámica y pruebas de laboratorio de un paciente en postoperatorio de un cambio valvular aórtico con hipertensión pulmonar (HP), complicado con EP (síndrome alveolo-intersticial). El interés de la presente comunicación es el de enfatizar el valor de la imagen por USG-P del EP (síndrome alveolo-intersticial).

Paciente masculino de 71 años de edad, con antecedente previo de hipertensión arterial sistémica en tratamiento con losartán/hidroclorotiazida desde el año 2008. Etilismo crónico intenso, consumo mayor a $60 \mathrm{~g} / \mathrm{d}$ ía durante más de 20 años. Inició en 2017 con disnea progresiva de grandes a moderados esfuerzos que limitó sus actividades diarias. Fue valorado en el medio hospitalario y tratado por insuficiencia cardíaca. En agosto de 2018, se documentó por ecocardiografía válvula aórtica calcificada, con fusión de comisuras, que condicionó doble lesión, estenosis grave e insuficiencia moderada con área valvular de $0.7 \mathrm{~cm}^{2}$, gradiente medio de $78.6 \mathrm{mmHg}$, velocidad máxima $6.5 \mathrm{~m} / \mathrm{s}$, presión sistólica de la arteria pulmonar $77 \mathrm{mmHg}$ y dilatación de la aurícula izquierda.

\section{Correspondencia:}

*Luis E. Santos-Martínez

Avenida Cuauhtémoc, 330

Col. Doctores, Del. Cuauhtémoc

Fecha de recepción: 24-02-2019

C.P. 06720, Ciudad de México, México

E-mail: sanlui@cardiologia.org.mx

1405-9940/○ 2019 Instituto Nacional de Cardiología Ignacio Chávez. Publicado por Permanyer. Este es un artículo open access bajo la licencia CC BY-NC-ND (http://creativecommons.org/licenses/by-nc-nd/4.0/).

Disponible en internet: 06-05-2019 Arch Cardiol Mex. 2019;89(4):408-411 www.archivoscardiologia.com
open access bajo la licencia CC 


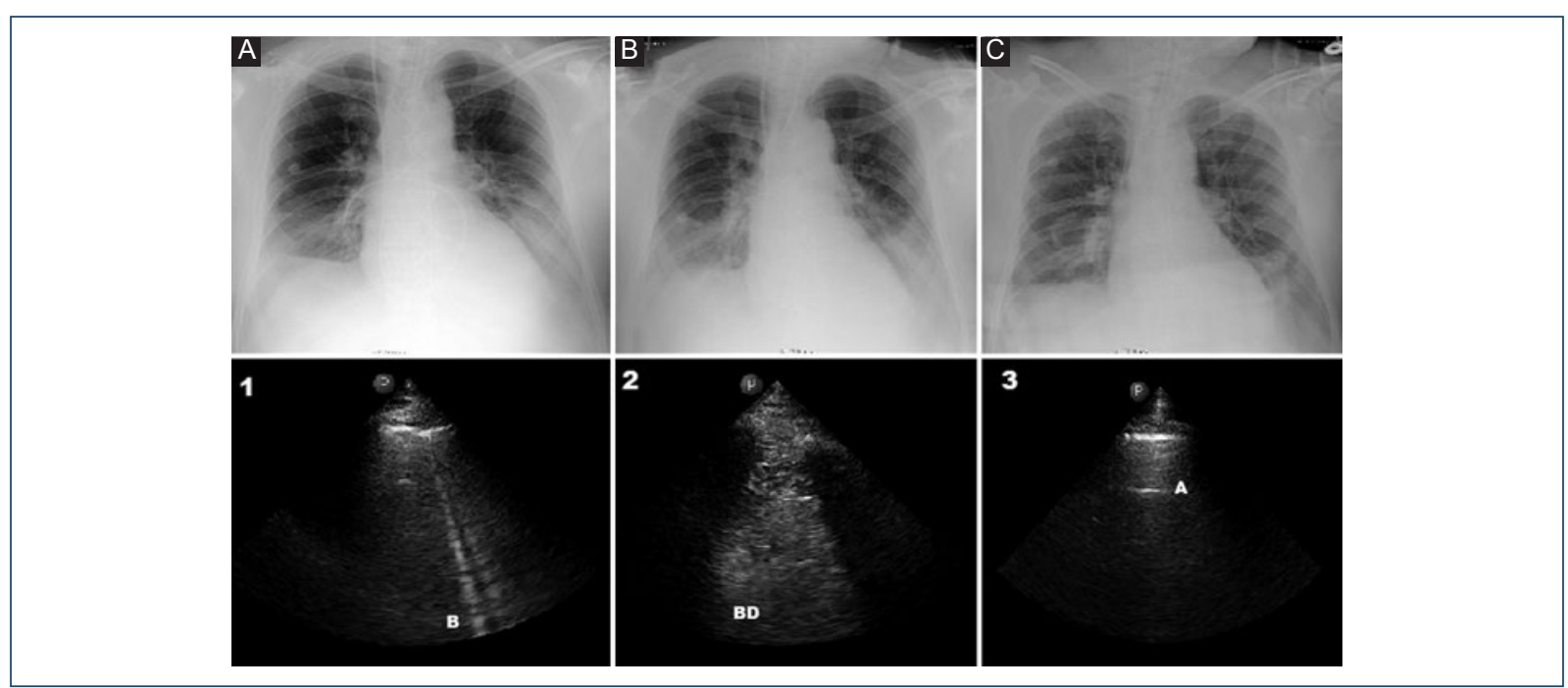

Figura 1. Correlación de la radiografía del tórax y el ultrasonido pulmonar en el paciente con síndrome alveolointersticial. Radiografía de tórax A: Con equipo portátil en anteroposterior, que sugiere presencia de ocupación intersticial y borramiento de ambos ángulos costo-diafragmáticos. Ultrasonido pulmonar 1: presencia de líneas B que sugiere ocupación intersticial (síndrome alveolo-intersticial). Radiografía de tórax B: Presencia de consolidación bilateral y borramiento de ambos ángulos costo-diafragmáticos, con incremento de la trama broncovascular. Ultrasonido pulmonar 2: evidencia de broncograma dinámico que sugiere consolidación. Radiografía de tórax C: Disminución del patrón de consolidación y mejoría en ambos ángulos costo-diafragmáticos. Ultrasonido pulmonar 3: disminución del patrón de broncograma dinámico y aparición de líneas A que sugiere en el ultrasonido un patrón normalizado.

Se operó en nuestra institución de cambio valvular aórtico, prótesis biológica Edwards \# 23 y plicatura de aorta ascendente. El tiempo quirúrgico fue de $314 \mathrm{~min}$, el tiempo anestésico de $390 \mathrm{~min}$, la circulación extracorpórea de $105 \mathrm{~min}$ y el pinzamiento aórtico de $88 \mathrm{~min}$. El sangrado total fue de $1060 \mathrm{ml}$, que se recuperó mediante recuperador celular, $962 \mathrm{ml}$. Se transfundieron 2 aféresis plaquetarias y $3 \mathrm{UI}$ de crioprecipitados, además de 1,000 UI de complejo protrombínico y $30 \mathrm{mcg} / \mathrm{i} . v$. de desmopresina $\mathrm{Al}$ egresar de quirófano el sangrado fue de $180 \mathrm{ml}$ en $50 \mathrm{~min}$.

Ya en la UCl, se complicó con sangrado mayor a lo habitual (420 ml/hora), por lo que se transfundió una unidad de paquete globular y $30 \mathrm{mcg}$ de desmopresina extra, y requirió reexploración quirúrgica en las primeras 4 horas.

Desarrolló síndrome de bajo gasto cardíaco (SBGC) (gasto cardíaco $3.4 \mathrm{l} / \mathrm{min}$, índice cardíaco $1.9 \mathrm{l} / \mathrm{min} / \mathrm{m}^{2}$, presión capilar pulmonar $7 \mathrm{mmHg}$, presión venosa central $10 \mathrm{mmHg}$, índice de resistencias vasculares sistémicas 1,105 dynas $/ \mathrm{m}^{2}$ ). Fue necesario un doble esquema vasopresor (norepinefrina, vasopresina) y fármaco con actividad inotrópica (dobutamina).

Presentó, además, deterioro en el intercambio gaseoso y mayor apoyo en la ventilación mecánica (fracción inspirada de oxígeno $80 \%$, presión positiva al final de la espiración $18 \mathrm{mmHg}$, presión arterial de oxígeno $51 \mathrm{mmHg}$, presión arterial de bióxido de carbono $37 \mathrm{mmHg}$, relación presión arterial de oxígeno/ fracción inspirada de oxígeno 63.7), por lo que se definió como la causa más probable el síndrome de dificultad respiratoria del adulto (SDRA) o, de acuerdo a su presentación por USG-P, como síndrome alveolo-intersticial. Su comportamiento por Rx de tórax y USG-P se muestra en la figura 1, además, en la tabla 1 se acotan las variables hemodinámicas, intercambio gaseoso, pruebas de laboratorio, ventilación mecánica y balance hídrico.

Este paciente, categorizado como del grupo II del consenso de HP, fue operado de cambio valvular aórtico ${ }^{8}$. La HP disminuyó una vez retirada la obstrucción valvular.

En el postoperatorio inmediato desarrolló SBGC y sangrado mayor al habitual. Tuvo deterioro cardiopulmonar, que en sujeto $>70$ años, con antecedentes de transfusiones e infección de vías aéreas inferiores, se catalogó como SDRA.

En este contexto, parece haber una sobreposición clínico-diagnóstica en un paciente en postoperatorio de cirugía cardíaca que presentó SBGC y que cursó con TRALI e infección de la vía aérea inferior, lo que condicionó el síndrome alveolo-intersticial, apoyado con 
Tabla 1. Comportamiento postoperatorio de las variables hemodinámicas, intercambio gaseoso, ventilación mecánica, balance hídrico y pruebas de laboratorio

\begin{tabular}{|c|c|c|c|}
\hline Variable & Día 1 & Día 2 & Día 3 \\
\hline $\begin{array}{l}\text { Hemodinámicas } \\
\text { Índice cardñiaco, } \mathrm{l} / \mathrm{min} / \mathrm{m}^{2} \\
\text { Gasto cardíaco, } \mathrm{l} / \mathrm{min} \\
\text { Presión venosa central, } \mathrm{mmHg} \\
\text { Presión capilar pulmonar, } \mathrm{mmHg} \\
\text { Presión media de la arteria pulmonar, } \mathrm{mmHg} \\
\text { Índice de resistencias vasculares sistémicas, dynas } / \mathrm{m}^{2}\end{array}$ & $\begin{array}{c}2.6 \\
4.7 \\
9 \\
11 \\
20 \\
1944\end{array}$ & $\begin{array}{c}2.8 \\
5.1 \\
6 \\
7 \\
18 \\
1696\end{array}$ & $\begin{array}{c}2.8 \\
5.2 \\
11 \\
13 \\
22 \\
2086\end{array}$ \\
\hline $\begin{array}{l}\text { Intercambio gaseoso arterial y venoso } \\
\text { Potencial hidrógeno arterial (pH) } \\
\text { Presión arterial de oxígeno, } \mathrm{mmHg} \\
\text { Presión arterial de bióxido de carbono, } \mathrm{mmHg} \\
\text { Bicarbonato sérico, mmol/l } \\
\text { Exceso de base, } \mathrm{mmol} / \mathrm{l} \\
\text { Saturación arterial de oxígeno, \% } \\
\text { Lactato sérico, } \mathrm{mmol} / \mathrm{l} \\
\text { Potencial hidrógeno venoso central (pH) } \\
\text { Presión venosa central de bióxido de carbono, } \mathrm{mmHg} \\
\text { Saturación venosa central de oxígeno, \% }\end{array}$ & $\begin{array}{c}7.42 \\
51 \\
37 \\
24 \\
-0.5 \\
88.7 \\
3.4 \\
7.28 \\
54 \\
56.8\end{array}$ & $\begin{array}{c}7.48 \\
69 \\
38 \\
27 \\
3 \\
95 \\
2 \\
7.44 \\
39 \\
68.9\end{array}$ & $\begin{array}{c}7.51 \\
66 \\
36 \\
29 \\
6.4 \\
95.3 \\
1.6 \\
7.5 \\
30.8 \\
55.7\end{array}$ \\
\hline $\begin{array}{l}\text { Ventilación mecánica } \\
\text { Presión positiva al final de la espiración, } \mathrm{cmH}_{2} \mathrm{O} \\
\text { Fracción inspirada de oxígeno, \% } \\
\text { Distensibilidad estática } \\
\text { Presión arterial de oxígeno/Fracción inspirada de oxígeno }\end{array}$ & $\begin{array}{c}8 \\
85 \\
49 \\
60\end{array}$ & $\begin{array}{c}10 \\
45 \\
70 \\
153\end{array}$ & $\begin{array}{c}9 \\
40 \\
69 \\
165\end{array}$ \\
\hline $\begin{array}{l}\text { Laboratorios } \\
\text { Hemoglobina, } \mathrm{mg} / \mathrm{dl} \\
\text { Leucocitos, } 10^{*} 3 / \mathrm{l} \\
\text { Neutrófilos totales, } 10^{*} 3 / \mathrm{l} \\
\text { Glucosa, } \mathrm{mg} / \mathrm{dl} \\
\text { Nitrógeno ureico (BUN), } \mathrm{mg} / \mathrm{dl} \\
\text { Creatinina sérica, } \mathrm{mg} / \mathrm{dl} \\
\text { Proteína C reactiva de alta sensibilidad, mg/dl } \\
\text { Balance hídrico, ml } \\
\text { Cultivo secreción bronquial }\end{array}$ & $\begin{array}{c}7.9 \\
17 \\
14.2 \\
157 \\
12 \\
1.4 \\
+4869\end{array}$ & $\begin{array}{c}8.8 \\
23 \\
21 \\
207 \\
38 \\
1.6 \\
61.32 \\
+5691 \\
\text { Bacilos gramnegativos } \\
\text { Cocos grampositivos }\end{array}$ & $\begin{array}{c}8.3 \\
24.1 \\
20.8 \\
106 \\
42 \\
1.69 \\
42 \\
+4715 \\
\text { Citrobacter } \\
\text { Proteus } \\
\text { Enterobacter }\end{array}$ \\
\hline
\end{tabular}

los valores de la citometría y los cultivos positivos, como puede observarse en la tabla 1 y la figura 1.

EI USG-P se ha convertido en una herramienta diagnóstica muy útil, dado que poder obtener imágenes en tiempo real es no invasivo, y no se necesita movilizar al paciente fuera de la $\mathrm{UCl}$.

El contraste de aire y agua condiciona valores de impedancia acústica diferente, lo cual ocasiona la reverberación, que puede verse en la pantalla como líneas verticales conocidas como líneas $\mathrm{B}^{2}$. Conforme el contenido de agua incrementa y el aire alveolar disminuye, esto es, se engrosa el septo interlobular y el espacio alveolar se llena de líquido, causa la aparición de múltiples y difusas líneas $\mathrm{B}^{2}$, el síndrome alveolo-intersticial. La sensibilidad diagnóstica ha sido reportada del $93.4 \%$ y especificidad $93 \%$.
En pacientes de cirugía cardíaca, se definió que el número de líneas $\mathrm{B}$ estaba relacionado con la cantidad de agua pulmonar extravascular (APEV) ${ }^{10}$. En falla cardíaca aguda, la cantidad de APEV se ha relacionado a mortalidad $^{10}$.

En otros tenores, la utilidad del USG-P podría ser de apoyo en unidades de urgencias para el diagnóstico diferencial de la disnea y ayudaría a distinguir las causas cardíacas de las pulmonares, como en la exacerbación de la enfermedad pulmonar obstructiva crónica, más aún, en la respuesta a tratamiento de la falla cardíaca aguda y a hemodiálisis en el paciente con EP2-4.

En cuanto a la neumonía, se demostró que el USG-P es confiable, en un estudio con 100 pacientes en la UCI con sospecha de neumonía se les realizó USG-P, $\mathrm{Rx}$ de tórax y tomografía computada. La sensibilidad 
(S), especificidad (E) valor predictivo positivo (VPP), valor predictivo negativo (VPN), así como la exactitud diagnóstica (EDx) respectivamente para el USG vs. Rx de tórax, fueron: S 97.5 vs.77.5, E 75 vs.60, VPP 94 vs. 88.6, VPN 88.2 vs. 40 , y EDx 93 vs. $74^{11}$.

Dos grandes limitaciones pueden verse en la intervención con USG-P en el sujeto en postoperatorio de cirugía cardíaca. La primera es en el diagnóstico diferencial entre el EP cardiogénico y no cardiogénico, ya que el USG-P solo mostrará la presencia de líneas B (síndrome alveolar-intersticial), y para el diagnóstico diferencial requerirá, además, de la evaluación hemodinámica (catéter de Swan-Ganz), como se señaló en este caso clínico. La otra gran limitación es la variabilidad inter e intraobservador, que podría tenerse con dste método diagnóstico ${ }^{2-4}$.

Desde el punto de vista del médico con especialidad en medicina crítica, el USG-P es una herramienta útil para el diagnóstico y monitoreo de las enfermedades del tórax en pacientes en condición crítica. A favor tiene el poder ser aplicado en tiempo real, en la cama del paciente, y libre de los riesgos de la radiación. Sus limitaciones serían el ser altamente dependiente del operador, la presencia de enfisema subcutáneo, compresas en el tórax o calcificaciones pleurales ${ }^{11}$.

Finalmente, el USG-P es una herramienta útil que apoya el diagnóstico y seguimiento de algunas entidades cardiopulmonares que acontecen en una población de pacientes que en el postoperio de cirugía cardiovascular.

\section{Financiación}

Ninguna.

\section{Conflicto de intereses}

Ninguno.

\section{Responsabilidades éticas}

Protección de personas y animales. Los autores declaran que para esta investigación no se han realizado experimentos en seres humanos ni en animales.

Confidencialidad de los datos. Los autores declaran que han seguido los protocolos de su centro de trabajo sobre la publicación de datos de pacientes.

Derecho a la privacidad y consentimiento informado. Los autores declaran que en este artículo no aparecen datos de pacientes.

\section{Bibliografía}

1. Shrestha GS, Weeratunga D, Baker K. Point of care lung ultrasound in critically ill patients. Rev Recent Clin Trials. 2018; 31;13(1):15-26.

2. Volpicelli G, Melniker LA, Cardinale L, Lamorte A, Frascisco MF. Lung ultrasound in diagnosing and monitoring pulmonary interstitial fluid. Radiol Med. 2013;118(2);196-205.

3. Cortellaro F, Ceriani E, Spinelli M, Campanella C, Bossi I, Coen D, et al. Lung ultrasound for monitoring cardiogenic pulmonary edema. Intern Emerg Med. 2016;12(7):1011-7.

4. Gargani L. Lung ultrasound: a new tool for the cardiologist. Cardiovascular Ultrasound. 2011;9:6

5. Rawal G, Yadav S, Kumar E. Acute respiratory distress syndrome: An update and review. J Transl Intern Med. 2018:6(2);74-7.

6. Reyes-Sánchez ME, Carrillo-Rojas JA, Hernández-Mercado MA, Amaro-Camacho JA, Herrera-Garza EH, López Pineda DM, et al. Síndrome de bajo gasto cardiaco post-cardiotomía. Arch Cardiol Mex. 2011;81(2):30-40.

7. Otrock ZK, Liu C, Grossman BJ. Transfusion』related acute lung injury risk mitigation: an update. Vox Sang. 2017;112(8):694-703.

8. Guazzi M, Galiè N. Pulmonary hypertension in left heart disease. Eur Respir Rev. 2012;1;21(126):338-46

9. Karim A, Arora VK. Applications of ultrasonography in respiratory care. Indian J Chest Dis Allied Sci. 2014;56(1); 27-31.

10. Frassi F, Gargani L, Tesorio P, Raciti M, Mottola G, Picano E, et al. Prognostic value of extravascular lung water assessed with ultrasound lung comets by chest sonography in patients with dyspnea and/or chest pain. J Cardiac Fail. 2007;13:830-5.

11. Nafae R, Eman SR, Mohamad NA, El-Ghamry R and Ragheb AS. Adjuvant role of lung ultrasound in the diagnosis of pneumonia in intensive care unit-patients. Egypt J Chest Dis Tuberc. 2013;62: 281-85. 